\title{
Intra-abdominal Hemorrhage Due to Rupture of a Mycotic Jejunal Artery Pseudoaneurysm in a 37-year-old Patient with Klebsiella Pneumoniae Endocarditis on Prosthetic Cardiac Valves: A Case Report
}

Ana-Maria Serban', Elena Andrei², Catalina Ionita ${ }^{2}$, Alexandru Martiniuc ${ }^{1,2}$, Marian Tudoroiu $^{3}$, Ovidiu Stiru ${ }^{24}$, Adrian Marinescu $^{5}$, Traian Dumitrascu ${ }^{1,2}$

Corresponding author:

Traian Dumitrascu, MD

Carol Davila University of Medicine and Pharmacy, Center of General

Surgery and Liver Transplant

Fundeni Clinical Institute

Fundeni Street no 258, 022328

Bucharest, Romania

E-mail: traian.dumitrascu@umfcd.ro
${ }^{1}$ Center of General Surgery and Liver Transplant, Fundeni Clinical Institute, Bucharest, Romania

${ }^{2}$ Carol Davila University of Medicine and Pharmacy, Bucharest, Romania

${ }^{3}$ Department of Anesthesiology and Intensive Care Medicine, Fundeni Clinical Institute, Bucharest, Romania

${ }^{4}$ Department of Cardiac Surgery, C.C. Iliescu Institute of Cardiovascular Diseases,

Bucharest, Romania

${ }^{5}$ Department of Infectious Diseases, Matei Bals Institute of Infectious Diseases, Bucharest, Romania

\section{ABSTRACT}

Visceral artery aneurysms (VAAs) are defined as aneurysms affecting the celiac, superior, or inferior mesenteric arteries and their branches. The incidence rate of aneurysms of superior mesenteric artery (SMA) and its branches is exceptionally infrequent, with only a handful of cases ever reported. Mycotic aneurysms (MA) in the context of infective endocarditis (IE) are also uncommon, particularly for Klebsiella Pneumonie species etiology. Hereby, it is presented a case of a 37-year-old male with previous prosthetic heart valves for IE who developed recurrent IE and furthermore presents with intra-abdominal hemorrhage due to a ruptured jejunal artery MA with Klebsiella Pneumoniae. The patient was surgically managed with resection of the MA and segmental bowel resection with anastomosis. The postoperative outcome was uneventful. Mycotic jejunal artery pseudoaneurysm in the context of IE on prosthetic heart valves is an exceptional appearance, particularly with Klebsiella Pneumoniae spp. etiology. In case of ruptured mycotic jejunal artery pseudoaneurysm surgery is usually required, particularly in the context of hemoperitoneum or intestinal ischemia.

Key words: jejunal artery pseudoaneurysm; mycotic aneurysm; infective endocarditis, prosthetic cardiac valves; hemorrhage; surgery

\section{INTRODUCTION}

Visceral artery aneurysms (VAAs) are defined as aneurysms affecting the celiac, superior, or inferior mesenteric arteries and their branches. VAAs include both true aneurysms (i.e., all three layers of the arterial wall are intact) and 
pseudoaneurysms (i.e., lack of a complete arterial wall), and are relatively rare (1).

The clinical features of a symptomatic disease differ for each anatomic location; however, clinical symptoms and signs are nevertheless nonspecific. VAAs are often not suspected initially in patients presenting with abdominal complaints, due to their rarity, which can lead to a delay in diagnosis. Consequently, these often present with life-threatening hemorrhage due to a high incidence of rupture $(2,3)$.

Thus, the general approach to VAAs is early elective intervention rather than watchful waiting, to minimize the risk of rupture. Management of VAAs depends mainly on the location of an aneurysm and the hemodynamic status of the patient. Differentiation of true aneurysms from pseudoaneurysms is also essential for the treatment approach. The most frequent location for VAAs is splenic artery (60\% - $80 \%$ of cases) and hepatic artery ( $20 \%$ of cases)(1). The incidence rate for jejunal artery pseudo-aneurysm is exceptionally infrequent, with only a handful of cases ever reported (4).

Preexisting aneurysms can become secondarily infected, but aneurysmal degeneration of the arterial wall can also be the result of infection that may be due to bacteremia or septic embolization, as in the case of mycotic aneurysms (MA) (associated with bacterial endocarditis), aneurysms that develop when material originating in the heart causes arterial wall infection and, subsequently, dilation (5).

Hereby it is presented the diagnosis and management of a patient with prior prosthetic cardiac valves for infective endocarditis (IE), with recurrent IE, and development of a ruptured mycotic jejunal artery pseudoaneurysm.

\section{CASE PRESENTATION}

A 37-year-old male was referred to our Department of Surgery in February 2018 by transfer from an Infectious Diseases Hospital for further investigation of a diffuse abdominal pain of 48 hours onset and febrile syndrome of two weeks onset.

His medical history included IE with no identified germs, severe aortic, mitral and tricuspid valve failure, severe secondary pulmonary hypertension, congestive heart failure NYHA Class III for which he has undergone cardiac surgery in August 2017 with the following procedures: aortic and mitral valve replacement with mechanical valve, De Vega tricuspid annuloplasty. The postoperative outcome was uneventful, with no signs of abnormalities at scheduled controls. However, in February 2018 he was admitted in the Infectious
Diseases Department for the persistent febrile syndrome. Blood cultures were positive for Klebsiella Pneumoniae spp.; therefore the patient received a complex antibiotics therapy regimen. The transthoracic and transesophageal echocardiography revealed a 1.8/ $0.7 \mathrm{~cm}$ mass with an irregular shape adherent to the mechanical mitral valve, highly suggestive of IE (fig. 1). No functional abnormalities were observed for the prosthetic aortic and mitral valves and no signs of pulmonary hypertension. The heart ejection fraction was $60 \%$. At the time of admission in our Department, the patient was hemodynamically, and respiratory stable and the clinical examination revealed abdominal distension, diffuse abdominal tenderness, with no signs of peritoneal irritation. Bioumoral tests revealed only elevated leukocytes $\left(42000 / \mathrm{mm}^{3}\right)$, mild anemia (hemoglobin serum level of $9.1 \mathrm{~g} / \mathrm{dL}$; normal range $13.5-17.1 \mathrm{~g} / \mathrm{dL})$, increased blood urea level $(79 \mathrm{mg} / \mathrm{dL}$; normal range $20-40 \mathrm{mg} / \mathrm{dL}$ ), and elevated fibrinogen serum level ( $418 \mathrm{mg} / \mathrm{dL}$; normal range $150-350 \mathrm{mg} / \mathrm{dL}$ ).

Contrast-enhanced computed tomography revealed a well-defined hyperdense mass of $3.8 / 4 \mathrm{~cm}$ in the root of the mesentery with surrounding leaked hematoma, highly suggestive of a superior mesenteric artery (SMA) branch pseudoaneurysm, with inside vegetations. Blood clots were also observed between the bowels and in perihepatic, perisplenic and pelvic space (fig. 2). Furthermore, multiple hypodense areas were observed in the spleen, with the maximum diameter of $3.1 \mathrm{~cm}$, suggestive of splenic infarctizations (fig. 3). Noteworthy, few jejunum loops were dilated with a thick wall (fig. 2).

An emergent midline laparotomy was performed revealing a ruptured jejunal artery pseudoaneurysm with secondary hemoperitoneum and large hematoma

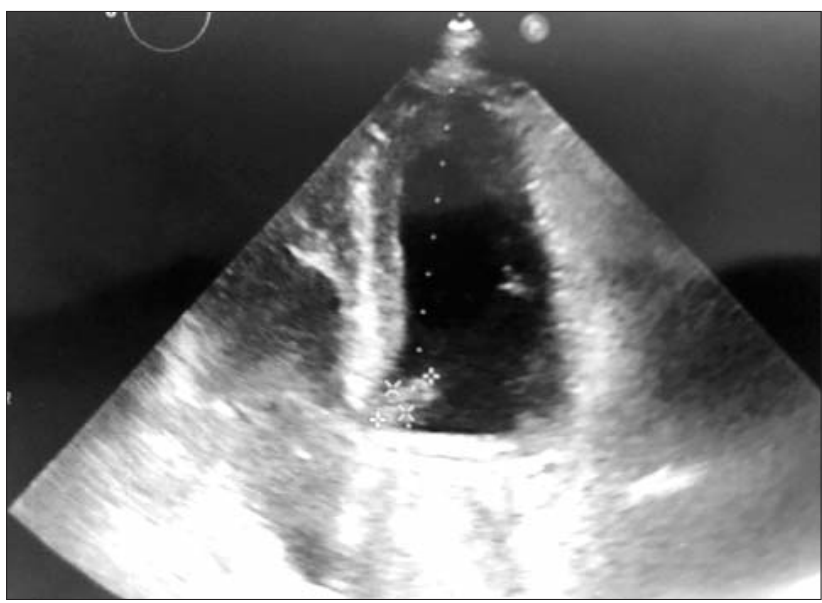

Figure 1 - Transthoracic echocardiography revealing a $1.8 / 0.7 \mathrm{~cm}$ mass $\left({ }^{*}\right)$ with an irregular shape adherent to the mechanical mitral valve 


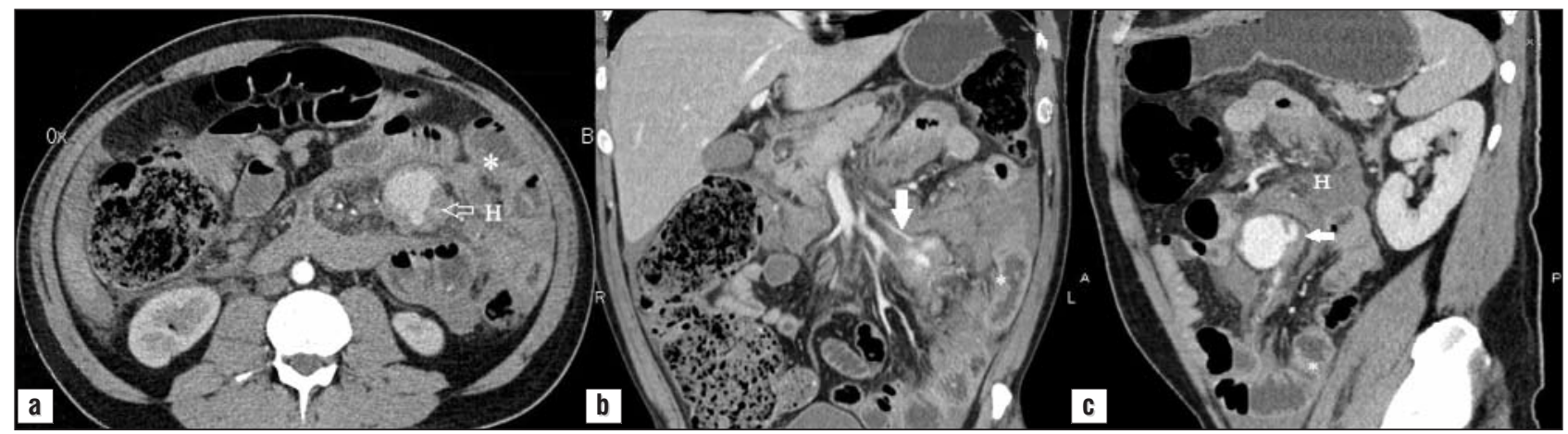

Figure 2 - Contrast-enhanced computed tomography (a) axial plane, arterial phase revealing a well-defined hyperdense mass of 3.8/ 4 $\mathrm{cm}$ in the root of the mesentery with surrounding leaked hematoma $(\mathrm{H})$, highly suggestive of a superior mesenteric artery (SMA) branch pseudoaneurysm, with inside vegetations and thrombus (white open arrow); (b) frontal plane, venous phase revealing feeding vessel (white filled arrow) and contrast extravasations; (c) sagittal plane, venous phase revealing feeding vessel (white filled arrow) and leaked hematoma (H). Note few jejunum loops with a thick wall $\left({ }^{*}\right)$

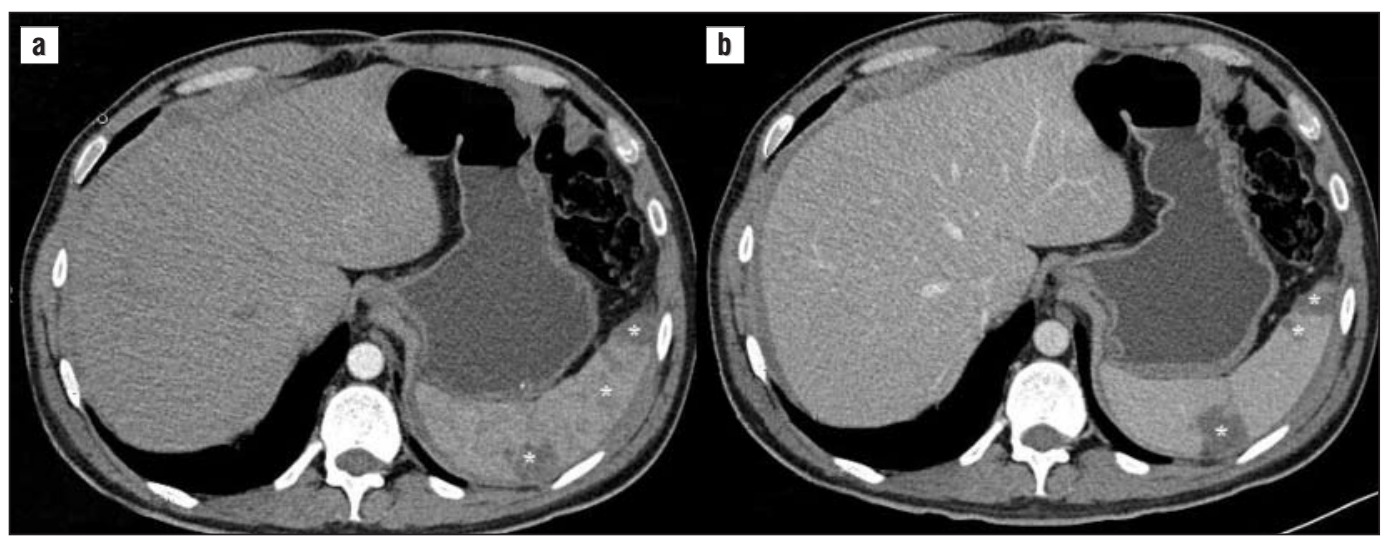

Figure 3 - Contrast-enhanced computed tomography axial plane, (a) arterial phase and (b) venous phase revealing multiple hypodense areas in the spleen $\left(^{*}\right)$, with the maximum diameter of $3.1 \mathrm{~cm}$, suggestive of splenic infarctizations

of mesentery root fused in the transverse mesocolon and the vicinity of the pancreas, and segmental intestinal ischemia (fig. 4). Inflammatory tumors of the spleen were also observed. The surgical procedure included resection of the arterial pseudoaneurysm, segmental jejunal enterectomy with end-to-end anastomosis, evacuation of the hematoma from the root of the mesentery and transverse mesocolon, splenectomy, peritoneal lavage and drainage (fig. 5).

The postoperative outcome was uneventful, with symptoms remittance, and the patient was transferred to the Infectious Diseases Department for further consolidation of the antibiotic therapy regimen of IE.

The pathology examination confirmed the diagnosis of arterial pseudoaneurysm with inside vegetations and ischemic lesions of the spleen (fig. 6).

\section{DISCUSSION}

Patients with prior IE and prosthetic heart valves are

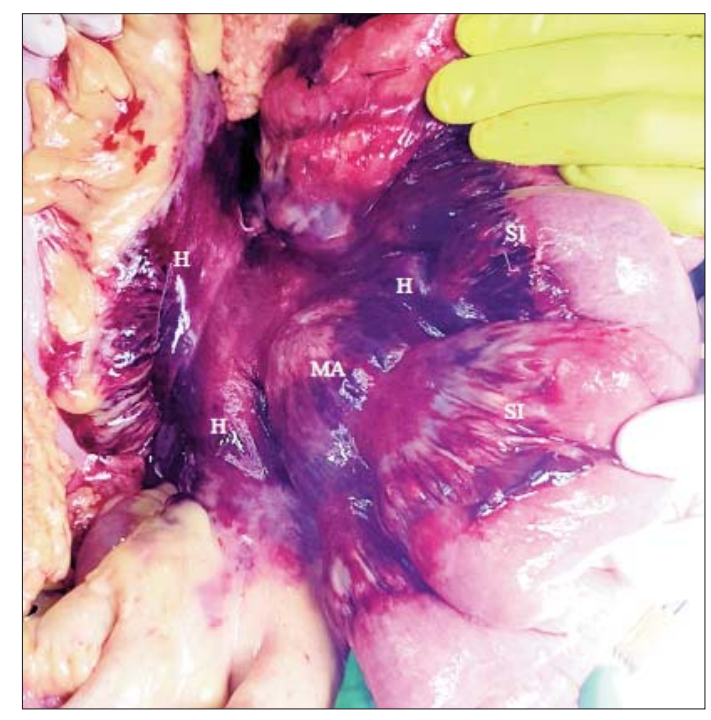

Figure 4 - Intraoperative aspects revealing a ruptured jejunal artery mycotic pseudoaneurysm (MA) with large hematoma of mesentery root fused in the transverse mesocolon and the vicinity of the pancreas $(\mathrm{H})$, and segmental intestinal ischemia (SI) 


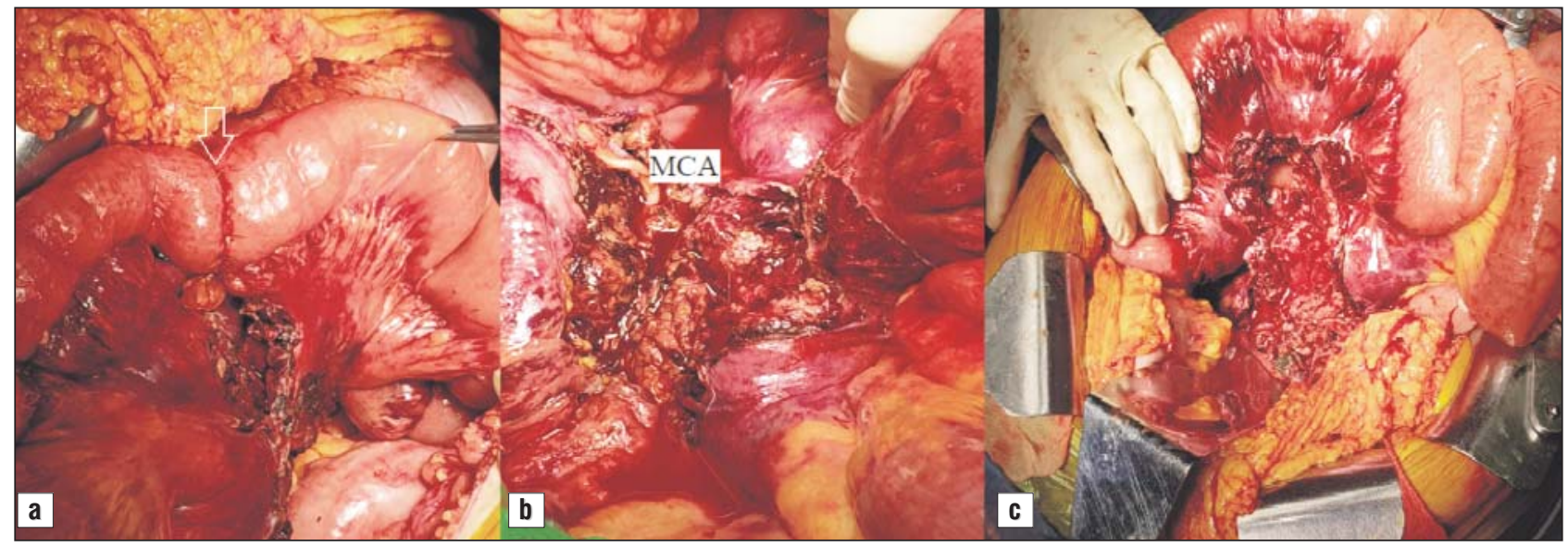

Figure 5 - Intraoperative aspects after resection of the arterial pseudoaneurysm, segmental jejunal enterectomy with end-to-end anastomosis (white arrow) (a), evacuation of the hematoma from the transverse mesocolon (b) and the root of the mesentery (c) (MCA - middle colic artery exposed)

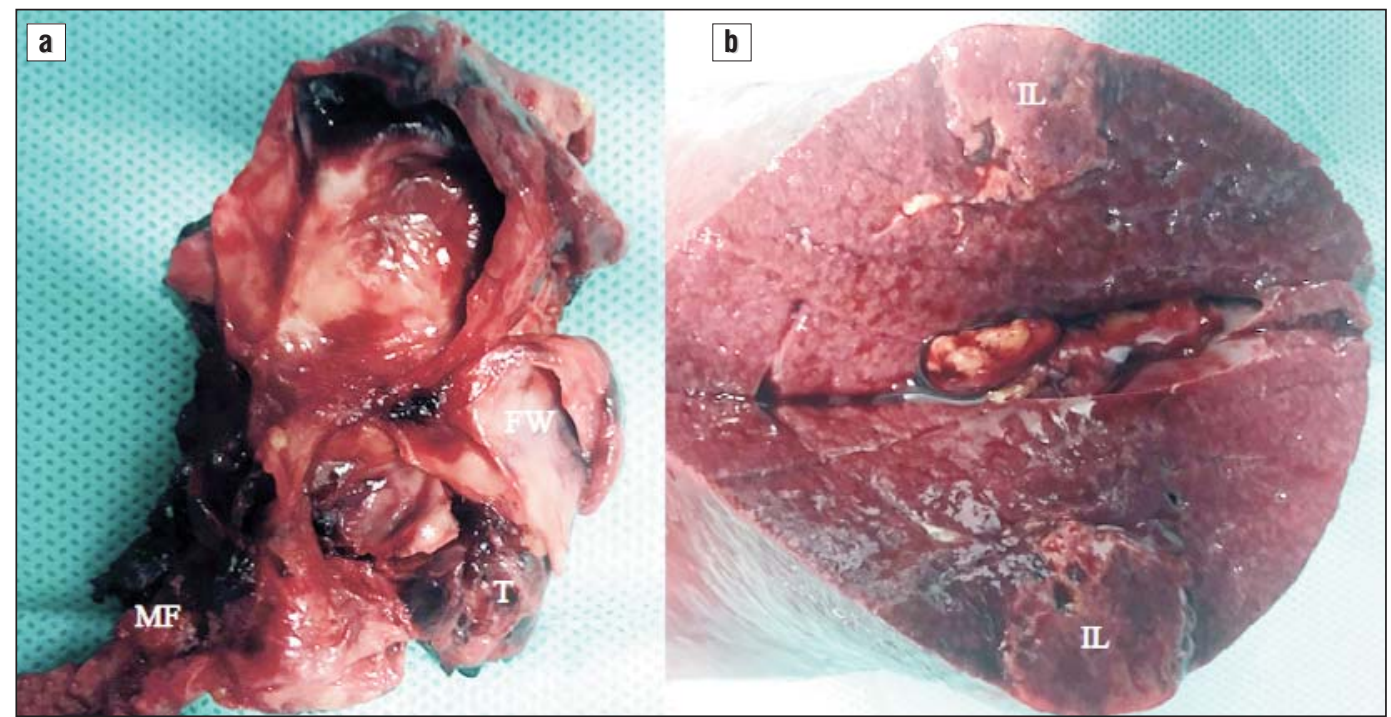

Figure 6 - Macroscopic aspect of the operative specimen (a) jejunal artery pseudoaneurysm with inside vegetations( ${ }^{*}$ ); Note mural thrombus (T), fibrous wall (FW)and surrounding mesenteric fat (MF);

(b) splenectomy with ischemic lesions (IL)

at high risk of developing IE (6), as it was the case in the present report. Gram-positive cocci are the primary etiologic germs for IE albeit sterile blood cultures are not uncommon when antibiotic therapy was administrated before sampling (7). Thus, Klebsiella Pneumoniae was rarely associated with IE (7). However, Klebsiella Pneumoniae is not an uncommon cause of MA, particularly in young patients with concurrent IE (8), as it was the case in the above-presented patient. Nevertheless, a MA rupture in Klebsiella endocarditis was previously reported in only one patient but with intracranial artery localization (9).

A MA is usually related with the aneurysms (true or false - pseudoaneurysm) caused by bacterial IE, albeit other causes were described (5).
Pathogenesis of MA includes septic microemboli to the vasa vasorum or septic embolus of a blood vessel, infection of the arterial wall from a contiguous dissemination, hematogenous infection during bacteriemia from a distant site or direct contamination of the artery (5). An embolism is estimated to occur in between $25 \%$ and $50 \%$ of patients with IE, but only about $1 \%$ to $5 \%$ develop symptomatic MA (10). Because of their embolic nature, MA tend to be multiple, but they can also be solitary (11). In the present case, septic emboli were observed in both jejunal artery and spleen.

MA can develop anywhere but are most commonly seen in the visceral arteries, followed by upper or lower extremity arteries and intracranial arteries (5). Other studies reported visceral arteries as the fourth cause of 
MA (12). Staphylococcus and Streptococcus species are the most common germs involved in MA (12).

Visceral artery MA represent less than $1 \%$ of intraabdominal MA (5). Furthermore, a review of the literature performed in 2016 identifies only 24 patients with SMA or its branches MA in the context of IE (13). These patients were predominantly young males, and surgery was the primary treatment approach $(13,14)$. Abdominal pain and fever are the most common presenting symptoms of SMA MA $(14,15)$. Noteworthy is that antibiotic therapy does not prevent the rupture of an MA of the SMA or its branches (16).

MA is widely considered a severe clinical condition, associated with a high risk of rupture and significant morbidity and mortality $(5,12,13)$. Thus, the rupture risk in SMA MA is higher than other visceral arteries MA and can lead to hemoperitoneum, mesenteric hematoma or gastrointestinal bleeding (15). Contrast-enhanced computed tomography represents the primary tool of diagnosis for patients with visceral artery MA $(5,12)$.

So far, the management of visceral artery MA is primarily based upon clinical experience guided by small case series. Although there are no consensus guidelines for optimal management of visceral artery MA, however, the standard treatment of these aneurysms is antibiotic therapy combined with either surgery or endovascular treatment, with or without revascularization (5). The specific approach of MA depends on the location and morphology of the VAA, presence and extent of hemorrhage, the presence of bowel ischemia, patient factors, and available expertise (12).

Endovascular therapy may be most suitable for the management of visceral artery MA in high-risk patients for whom mortality rates for open surgery would be prohibitive (15). Endovascular management may also be beneficial for patients with aneurysm rupture, as a temporizing measure, until definitive debridement and reconstruction can be performed under more "elective" circumstances (17). Nevertheless, open surgical repair remains the preferred option to treat SMA and its branches MA (15).

In the above-mentioned patient, the surgical approach was mandatory due to the rupture of the MA pseudoaneurysm with secondary hematoma, hemoperitoneum, and intestinal ischemia. Concomitant small bowel resection for ischemia was reported in more than one-third of patients with SMA and its branches MA (15).

\section{CONCLUSION}

Mycotic jejunal artery pseudoaneurysm in the context of IE on prosthetic heart valves is an exceptional appearance, particularly of Klebsiella Pneumoniae spp. etiology. For these particular cases, both diagnostic and management are sometimes challenging. Presence of abdominal pain in a patient with documented IE should raise the potential diagnosis of a visceral artery MA and further exploration to rule out or to confirm the diagnosis is required. In case of ruptured mycotic jejunal artery pseudoaneurysm surgery is usually required, particularly in the presence of hemoperitoneum or intestinal ischemia.

\section{REFERENCES}

1. Nosher JL, Chung J, Brevetti LS, Graham AM, Siegel RL. Visceral and renal artery aneurysms: a pictorial essay on endovascular therapy. Radiographics 2006;26(6):1687-704.

2. Etezadi V, Gandhi RT, Benenati JF, Rochon P, Gordon M, Benenati $\mathrm{MJ}$, et al. Endovascular treatment of visceral and renal artery aneurysms. J Vasc Interv Radiol 2011;22(9):1246-53.

3. Ferrero E, Ferri M, Viazzo A, Robaldo A, Carbonatto P, Pecchio A, et al. Visceral artery aneurysms, an experience on 32 cases in a single center: treatment from surgery to multilayer stent. Ann Vasc Surg 2011;25(7):923-35

4. Asano $M$, Nushida $H$, Nagasaki $Y$, Tatsuno $Y$, Ueno $Y$. Rupture of a jejunal artery aneurysm. Leg Med (Tokyo) 2008;10(5):268-73.

5. Wilson WR, Bower TC, Creager MA, Amin-Hanjani S, O'Gara PT, Lockhart PB, et al. Vascular Graft Infections, Mycotic Aneurysms, and Endovascular Infections: A Scientific Statement From the American Heart Association. Circulation. 2016;134(20):e412-e460.

6. Ostergaard L, Valeur N, Ihlemann N, Smerup MH, Bundgaard H, Gislason $\mathrm{G}$, et al. Incidence and factors associated with infective endocarditis in patients undergoing left-sided heart valve replacement. Eur Heart J 2018;39(28):2668-75.

7. Brouqui $P$, Raoult $D$. Endocarditis due to rare and fastidious bacteria. Clin Microbiol Rev 2001;14(1):177-207.

8. Hsu PJ, Lee CH, Lee FY, Liu JW. Clinical and microbiological characteristics of mycotic aneurysms in a medical center in southern Taiwan. J Microbiol Immunol Infect 2008;41(4):318-24.

9. Pillai SM, Bharadwaj VK. Mycotic aneurysm rupture in Klebsiella endocarditis. Indian Pediatr 2014;51(4):323-4.

10. Baddour LM, Wilson WR, Bayer AS, Fowler VG, Jr., Tleyjeh IM, Rybak MJ, et al. Infective Endocarditis in Adults: Diagnosis, Antimicrobial Therapy, and Management of Complications: A Scientific Statement for Healthcare Professionals From the American Heart Association. Circulation 2015;132(15):1435-86.

11. Gonzalez I, Sarria C, Lopez J, Vilacosta I, San RA, Olmos C, et al. Symptomatic peripheral mycotic aneurysms due to infective endocarditis: a contemporary profile. Medicine (Baltimore) 2014;93(1): 42-52.

12. Lee WK, Mossop PJ, Little AF, Fitt GJ, Vrazas JI, Hoang JK, et al. Infected (mycotic) aneurysms: spectrum of imaging appearances and management. Radiographics 2008;28(7):1853-68.

13. De Troia A, Mottini F, Biasi L, Azzarone M, Tecchio T, Salcuni P Superior Mesenteric Artery Aneurysm Caused by Aortic Valve Endocarditis: The Case Report and Review of the Literature. Vasc Endovascular Surg 2016;50(2):88-93.

14. Kordzadeh A, Watson J, Panayiotopolous YP. Mycotic aneurysm of the superior and inferior mesenteric artery. J Vasc Surg 2016;63(6): 1638-46.

15. Sharma G, Semel ME, McGillicuddy EA, Ho KJ, Menard MT, Gates JD. Ruptured and unruptured mycotic superior mesenteric artery aneurysms. Ann Vasc Surg 2014;28(8):1931-8.

16. Li YT, Liu XB, Wang T. Antibiotic therapy did not prevent the rupture of mycotic aneurysm of the superior mesenteric artery. Heart Surg Forum 2015;18(3):E088-E089.

17. Klonaris C, Katsargyris A, Vasileiou I, Markatis F, Liapis CD, Bastounis E. Hybrid repair of ruptured infected anastomotic femoral pseudoaneurysms: Emergent stent-graft implantation and secondary surgical debridement. J Vasc Surg 2009;49(4):938-45. 